\title{
Application Progress of OCTA in Retinal Diseases
}

\author{
Sun Shanshan ${ }^{1, *}$, He Guiyun ${ }^{2}$ \\ ${ }^{1}$ Department of Medical College, Hunan Provincial People's Hospital, The First Affiliated Hospital of Hunan Normal University, Changsha, \\ China \\ ${ }^{2}$ Hunan Provincial People's Hospital, Changsha, China
}

Email address:

653952692@qq.com (Sun Shanshan),1747768543@qq.com (He Guiyun)

\section{To cite this article:}

Sun Shanshan, He Guiyun. Application Progress of OCTA in Retinal Diseases. International Journal of Ophthalmology \& Visual Science. Vol. 6, No. 2, 2021, pp. 80-88. doi: 10.11648/j.ijovs.20210602.14

Received: March 23, 2021; Accepted: April 7, 2021; Published: April 16, 2021

\begin{abstract}
Optical coherence tomography angiography (OCTA), as a nascent fundus vascular angiography technique, can identify the blood flow movement information of the retina and choroid with high resolution, and imaging the microvascular circulation of the retina and choroid in living tissues. Compared to previous art dye-based imaging such as fluorescein angiography and indocyanine green angiography, it has the characteristics of fast, non-invasive, high resolution and three-dimensional imaging, as well as the ability to display retinal vascular structure and blood flow information simultaneously. OCTA is a nascent technology with a potential wide applicability for retinal vascular disease. As a part of systemic blood circulation, ocular blood flow has been affected by a variety of factors. Vascular factors play an important role in eye diseases including retinal vein occlusion, diabetic retinopathy and glaucoma, and other diseases. OCTA has special advantages in retinal choroidal vascular changes, disease management follow-up and treatment effect detection. However, OCTA suffers from disadvantages of a relatively small field of view, inability to show leakage, and proclivity for image artifact due to patient movement or blinking. This article will review the recent research of OCTA in diabetic retinopathy, retinal vein occlusion, primary glaucoma, age-related macular degeneration and high myopia fundus to understand its applicability in the research and clinical practice of retinal diseases.
\end{abstract}

Keywords: Optical Coherence Tomography Angiography, Vascular Density, Retinal Diseas, Diabetic Retinopathy

\section{Introduction}

Optical coherence tomography (OCT) was one of the biggest advances in ophthalmic imaging, which can finely visualize the interlayer structure of the retina. Since the retinal vascular information cannot be displayed, Fundus fluorescein angiography (FFA) is often used clinically to observe the retinal vascular structure. FFA imaging is invasive, requires intravenous contrast agents and cannot be observed at all levels of the retina. Basically only superficial capillary plexus can be seen. Nevertheless, FFA is still the gold standard for diagnosis of ophthalmic retinal diseases. In recent years, with the rapid development of science and technology, the diagnosis and treatment of ophthalmic diseases has become more and more accurate. As a new type of fundus vascular examination technology, OCTA provides the possibility of imaging the superficial capillary plexus as well as the middle and deep capillary plexus. OCTA can detect changes in choroidal blood vessel flow and can elucidate the presence of choroidal neovascularization $(\mathrm{CNV})$ in a variety of conditions. It provides a highly detailed view of the retinal vasculature, which allows for accurate delineation of the foveal avascular zone (FAZ) in diabetic eyes and detection of subtle microvascular abnormalities in diabetic and vascular occlusive eyes. Optic disc perfusion in glaucomatous eyes is notable as well on OCTA. OCTA can provide deep-resolved retinal and choroidal blood flow images with a level of detail far exceeding FFA. Although still in development, OCTA can produce images of blood flow that have unprecedented resolution of all the vascular layers of the retina in a rapid, non-invasive fashion. OCTA makes the description and quantification of the disease, the study of the pathogenesis of the disease, and the development and evaluation of new therapies become possible. To a great extent, it promotes the diagnosis, treatment and research level of clinical ophthalmic diseases. This paper will elaborate and summarize the clinical application of OCTA in ophthalmology. 


\section{The Working Principle of OCTA}

OCTA is a non-invasive imaging technology that uses motion contrast imaging to generate high-resolution vascular blood flow information. OCTA can also generate angiography images in seconds. Based on repeated B-scans from the same location on the retina, time signal changes caused by moving particles (such as red blood cells that flow through blood vessels) can be separated from other signal changes (eye movement or noise in the OCT signal). As a result, an image contrast can be formed between the moving blood vessels and the surrounding static tissue [1-3]. At present, the most widely used method is the split-spectrum amplitude-decorrelation angiography (SSADA), which is an improved amplitude-based angiographic method.

In SSADA, the illumination beam is divided into a plurality of spectral bands. This provides improved speckle contrast between the movement of red blood cells and the motion of adjacent structures. The traffic signal is enhanced and the background noise is reduced [4]. The acquired images are close to the resolution of histological level. Because of the simple, fast, non-invasive, high-resolution layered scanning imaging technology for OCTA, many basic and clinical research applications have become possible.

\section{The Difference Between FFA, ICGA and OCTA}

FFA is helpful to assess the retinal vasculature. It was first described in 1961 and was subsequently used as a standard operating method in the field of ophthalmology [5]. Indocyanine green angiography (ICGA) is a choroidal angiography technique that helps to assess the choroidal circulation and vasculature. Both FFA and ICGA are invasive tests, which require intravenous injection of dye for visualization, and the imaging time is as high as 10-30 minutes [6-9]. They provide a two-dimensional image set that can dynamically observe blood flow in a wide field of view. They have the ability to evaluate perfusion (such as retinal arteriovenous filling time), leakage and staining [10]. So far, FFA and ICGA are still the gold standard for the diagnosis of retinal diseases in ophthalmology. For example, it can be used to detect $\mathrm{CNV}$, retinal neovascularization (RNV), neovascularization of the optic disc and others [11-13]. Because it can only be developed by intravenous injection of dye, retinopathy may be covered by dye leakage and bleeding or media opacity. Since the imaging image provided is a two-dimensional image, it becomes difficult to determine the location of the depth of the lesion and the extent of neovascularization. FFA or ICGA usually cannot divide different retinal layers, therefore, determining the axial position of the pathology requires understanding the patterns of occlusion and leakage [14]. For example, to distinguish between the type $1 \mathrm{CNV}$ found between the retinal pigment epithelium (RPE) and Bruch's membrane and the type $2 \mathrm{CNV}$ found in the retinal space above the RPE, it is necessary to understand that RPE has a physiological barrier to fluorescence that prevents leakage. Therefore, the lesion site of type $1 \mathrm{CNV}$ requires a large amount of dye accumulation, and there will be obvious superfluorescence phenomenon [15]. FFA and ICGA have other shortcomings. They all need intravenous injection of fluorescein to visualize blood vessels. Although it has been proven to be safe, intravenous injection of dye is bound to face allergic reactions or systemic side effects [16-18]. For some special patients such as pregnant women or patients with abnormal renal function, their use will be restricted. In busy clinical work, it will also cause some pressure on doctors or patients because of its long imaging time. Some patients may need frequent follow-up observation to track the development of the disease, the patient may become intolerable. Thus rapid, non-invasive, visualization of the retina and choroidal blood vessels will be beneficial to the patient. In contrast, OCTA is a non-invasive technique that can obtain angiographic information without the use of dyes. In terms of speed and imaging information, OCTA has many advantages over dye angiography [19]. The retinal vascular structure can be visualized without dyes and the retina can be layered to obtain high-resolution images. The OCTA image is essentially a motion contrast image, which is obtained by performing multiple B-scans at the same position to obtain depth-resolved imaging of the retinal vasculature [20], It then generates images of the superficial and deep retinal layers [21], and these images can be modified to further subdivide the retinal vascular system to provide images at other levels, such as the outer retina and choroidal capillaries $[19,22,23]$. The image resolution of $3 \mathrm{~mm} \times 3 \mathrm{~mm}$ OCTA angiography images is higher than that of currently FFA or ICGA images. Matsunaga et al concluded that their ability to display important vascular details was equivalent [24]. OCTA can make the retina and choroidal microvasculature visible, which is conducive to our deep understanding of retinal diseases.

OCTA also has some disadvantages. First, the field of view of OCTA is narrower than that of FFA, and most images are 3 $\mathrm{mm} \times 3 \mathrm{~mm}$. Therefore, it is difficult for OCTA to produce high-resolution peripheral retinal images [19]. Second, OCTA is unable to evaluate the dynamic characteristics of velocity and leakage, which is necessary for evaluating various retinal pathologies. Third, processing of high-resolution images may be time-consuming [25]. Due to the existence of superficial blood flow, the images generated by OCTA are extremely susceptible to projection artifacts, making it difficult to interpret deep retinal blood vessels [26]. Although this can be corrected by the projection removal algorithm, this method may result in the loss of deeper internal flow information and image separation [27]. Fourth, the probability of artifacts (flicker, eye movement, blood vessel ghosting) in OCTA images increases. The artifacts may affect the interpretation of image results, and OCTA may not detect capillaries with flow rates lower than the detection threshold [28]. 


\section{The Application of OCTA in Retinal Diseases}

\subsection{Diabetic Retinopathy}

Diabetic retinopathy (DR) is still the main cause of visual impairment and blindness in the world. About 285 million people worldwide have diabetes. About $33 \%$ of people do not have any signs of diabetic retinopathy, but about $11 \%$ of people have vision-threatening diabetic retinopathy [29]. With the epidemic trend of obesity and changes in diet and exercise patterns, the number of patients who have diabetic retinopathy and are at risk of blindness will increase [30]. Early detection and accurate staging of diabetic retinopathy are of great significance for guiding the diagnosis and treatment of diabetic retinopathy. Studies have found that diabetic retinopathy mainly occurs 10 to 20 years after the diagnosis of type 2 diabetes, or 5 to 10 years after the diagnosis of type 1 diabetes [31]. Histological studies of human cadaver eyes and animal model eyes have shown that the pathological changes of DR occur many years before the clinical manifestations [32, 33]. Therefore, it is very likely that we will not find the disease until the relatively late stage of microvascular changes. In the relatively early stage of microvascular changes, these pathological changes cannot be detected clinically or on angiography.

OCTA is an emerging technology that can safely, quickly and non-invasively display the retinal microvasculature with a resolution exceeding FFA and close to histological accuracy. Therefore, OCTA provides clinicians with a way to detect ongoing subclinical microvascular changes in DR before clinical signs and symptoms occur. Recent OCTA studies have shown that the changes of retinal vessels in patients with DR have been qualitatively evaluated. OCTA can observe microcirculatory changes in early diabetic retinopathy. (1) Microaneurysm (MAs). MAs is a lesion that is usually shown in early DR. MAs are locally expanded cystic or fusiform capillary. It is shown as a high reflection spot around the FAZ on OCTA, and can be shown as various morphological MAs, including spindle, saccular, curved, and coiled shapes, while they can only show uniform strong fluorescent spots on FAA [34-36]. Peres et al. [37] studies have proved that the number of MAs in OCTA in shallow capillary plexus (SCP) and deep capillary plexus (DCP) is statistically different from that in FFA, and the number of MAs detected by OCTA in DCP is higher than that in SCP. The decrease of MAs in SCP and DCP is related to the better response of Diabetic macular edema (DME) to anti-VEGF [38], which may be beneficial to the observation of the therapeutic effect of anti-VEGF in patients with DME. (2) Neovascularization. OCTA can also distinguish the structural levels of angiopathy and uniquely distinguish retinal neovascularization from retinal vein occlusion or shunt vessels. To distinguish between optic disc neovascularization and optic disc vascular collateral, the former enters the vitreous body and forms a network of weak blood vessels, while the latter is a ring of small blood vessels [39-42]. The neovascularization of retina and optic disc is characterized by flower-shaped interwoven vessels on the surface of retina and optic nerve [35]. It is not always possible to distinguish intraretinal microvascular abnormality (IRMA) from RNV by clinical examination or FFA [35, 43-45]. About 50\% of RNV occurs near IRMA, which increases the diagnostic value of OCTA in distinguishing IRMA from RNV. In a series of studies, it was found that $92 \%$ of retinal neovascularization occurred in the non-perfusion area of retinal capillaries [46]. Therefore, OCTA may help to distinguish severe non-proliferative diabetic retinopathy from proliferative diabetic retinopathy, and may help to closely track severe non-proliferative diabetic retinopathy cases. The study of morphological clues in OCTA can reflect the function and activity of neovascularization, and can be used to grade vascular pathological activities, find treatment targets and track them. (3) FAZ: OCTA is considered to be superior to FFA in defining the microvascular system in the center of the macula, because it is not covered by dye-leaking fluorescein. FAZ is located in the center of the macula, a capillary-free area surrounded by the foveal capillary ring. Abnormal structure or perfusion in this area can severely affect vision. Normal FAZ refers to the round or oval shape of the vascularized area on the OCTA. Due to the loss of vascular integrity, FAZ in diabetic eyes will increase [44]. With the development of DR, the increase of FAZ may indicate the increase of non-perfusion area [47]. For example, studies have shown that the OCTA of 63 eyes of 63 patients showed a significant increase in FAZ, from 0.25 $\mathrm{mm}^{2}$ in the control group to $0.37 \mathrm{~mm}^{2}$ in diabetic eyes without DR and $0.38 \mathrm{~mm}^{2}$ in diabetic eyes with DR [48].

One of the most important applications of OCTA will detect subclinical changes in DR. However, judging from the current research situation, the changes of microvessels are beyond the range that can be detected by clinical examination at this stage. The non-invasive and rapid characteristics of OCTA make the fundus retinal imaging of patients more safe and convenient, and subtle, subclinical retinal microangiopathy is not uncommon in patients with diabetes. OCTA provides us with objective monitoring of these minor changes and provides evidence of the severity of DR. At present, there are a large number of studies on whether subclinical DR changes can actually be detected in OCTA, so as to provide help for early intervention and prevention of vision loss.

\subsection{Retinal Vein Occlusion}

Retinal vein occlusion (RVO) is the second most common retinal vascular disease and a common cause of vision loss in elderly patients [49]. There are two types of RVO: central retinal vein occlusion (CRVO) and branch retinal vein occlusion (BRVO). Central retinal vein occlusion is further divided into two categories: ischemic and non-ischemic. Non-ischemic CRVO is the most common, accounting for about $70 \%$ of cases. The best corrected visual acuity is usually better than 20/200 [50]. Ischemic CRVO may be the origin or progression of non-ischemic CRVO. The visual prognosis of 
ischemic CRVO is much lower, accounting for about $30 \%$ of cases. About $90 \%$ of patients with visual acuity less than $20 / 200$ have ischemic central retinal vein occlusion [51]. In the acute phase of CRVO, the evaluation of microvascular circulation and structure by FFA is limited due to bleeding and edema [52]. OCTA has recently been introduced as a non-invasive method to provide microvascular assessment $[53,54]$.

OCTA can assess the difference between ischemic and non-ischemic CRVO. The microvascular changes of CRVO patients on OCTA are mainly manifested in six characteristics: (1). Retinal non-perfusion area: the ability of the non-perfusion area of the retina shown by OCTA is similar to that of the non-perfusion area of the retina shown by FFA [55]. In FFA images, with the covering effect of fluorescein leakage in the middle and late stage of radiography, the boundary of the non-perfusion area is not clear. But OCTA can clearly show the boundary of the non-perfusion zone of the retina without being affected by the shaded fluorescence, and that non-perfusion area of the retina is mainly concentrated in the DCP [56]. OCTA can also distinguish the FAZ from the non-perfusion areas [57], and the area of the non-perfusion area can be quantified, so that the size of the no-perfusion area can be calculated accurately. It is worth noting that in the presence of cystoid macular edema (CME), the presence of edema will lead to a weakening of the scanning signal, and the results of imaging analysis may affect our assessment of the non-perfusion area of the retina [58]. The lack of visible blood vessels in the non-perfusion area may not be due to a complete lack of blood flow, but the blood flow may be reduced below the detection threshold of the device. (2). Decrease of vascular density: in terms of blood flow density, the blood vessel density of the fovea and parafoveal area and the entire scanning area of RVO eyes are lower than those of the control group [59-61]. (3). Neovascularization: retinal neovascularization can be induced in patients with ischemic RVO, OCTA is highly sensitive to the detection of neovascularization in the macular area and optic disc. However, OCTA itself has the limitation of small scanning range, so it is not conducive for us to observe the peripheral neovascularization of retina [57]. (4). The formation of collateral vessels: OCTA can also observe the formation of collateral vessels in superficial and deep vascular plexus in CRVO patients. Collateral circulation often occurs at the junction of occlusion veins and normal veins or veins that pass through non-perfusion areas. In the FFA results, if the eye has retinal hemorrhage or retinal edema, it is difficult for us to clearly observe these microvascular abnormalities. (5). Microaneurysms: for the observation of microaneurysms, it is impossible to observe which layer the microaneurysm is located in the FFA image. OCTA is more accurate than FFA in observing microhemangioma. In CRVO patients, microvessels were found to be more common in DCP than in SCP. It is usually formed in the boundary of the non-perfusion area and collateral vessels [56, 60]. (6). FAZ: the FAZ area measured by FFA images in healthy people is $0.205 \sim 0.405$ $\mathrm{mm}^{2}$, the FAZ area measured by OCTA is $0.25 \sim 0.30 \mathrm{~mm}^{2}$ in the superficial retina, and $0.4 \mathrm{~mm}^{2}$ in the deep retina $[59,62$,
63]. In RVO patients, FAZ was enlarged and deformed, and the destruction of FAZ in DCP is more common than in SCP [64]. The degree of enlargement in FAZ is used as a predictor of macular ischemia and final visual acuity in patients with RVO [65]. ADHI et al. [58] went through a prospective study of OCTA and found that the FAZ area of patients with RVO was larger than that of the contralateral eye of RVO patients and healthy control group. The FAZ area of the contralateral eye of RVO patients also increased compared with the healthy control group. This study suggests our OCTA can detect possible early pathological changes in early vascular occlusive disease.

\subsection{Primary Glaucoma}

Glaucoma is a group of diseases characterized by optic nerve atrophy and visual field defect. It is one of the major blindness diseases in the world. It has seriously threatened the visual health and visual quality of human beings. The incidence rate is increasing year by year. It is estimated that the number of glaucoma cases worldwide will exceed 110 million by 2040 [66]. For a long time, the changes of ocular perfusion are related to the pathogenesis of glaucoma, but few details are known. Current studies believe that the pathogenesis of induced glaucoma is mainly mechanical compression theory and vascular theory (microcirculation). More and more research evidences show the mechanism of microvascular dysfunction in the pathogenesis of glaucoma $[67,68]$. As a new blood flow imaging method, OCTA provides a new method for monitoring the early fundus damage in primary glaucoma, and reveals the pathogenesis of glaucoma at the blood flow level.

The preliminary study of OCTA was carried out in primary open angle glaucoma (POAG) eyes. The results showed that the blood flow index and vascular density of optic nerve and peripapillary area of POAG eyes were lower than those of the control group [69, 70]. And the vascular density of the superficial retina in the macular area of glaucoma also decreased [71, 72]. With the increase of the severity of glaucoma, the vascular density showed a more significant decrease [70, 73]. Recently, deep microvascular shedding (MvD) has been observed in the choroid of POAG eyes, which is defined as the loss of choroidal capillaries in the area of paranipple atrophy $[74,75]$. These changes on OCTA are closely related to the functional changes in visual field examination and the structural changes in optical coherence tomography (OCT), which are changes in the parapapillary retinal nerve fiber layer and changes in the thickness of the inner retinal layer [74, 76]. Mansoori et al. showed that the decrease of vascular density was mostly related to the severity of visual field damage, and it was found that the vascular density around the optic papilla decreased in the corresponding location of glaucoma visual field defect [77]. In addition, it was also found that the capillary density around the optic papilla in NTG eyes was significantly lower than that in POAG eyes, but the degree was different from that in POAG eyes.

Studies also found that the density of capillaries around the 
optic papilla of normal tension glaucoma (normaltension glaucoma, NTG) eyes is also significantly reduced, but the degree was different from that of POAG eyes [78]. The changes in vascular density observed by OCTA may actually occur before the decline in structure and function, so it may be useful for the diagnosis and monitoring of very early glaucoma [79].

Since OCTA is a recently developed new technology, research to evaluate the long-term progress of glaucoma is continuing. Current studies have shown that OCTA can detect a progressive decrease in superficial vascular density in glaucoma even if monitored in a short period of time [68, 80]. OCTA is a safe, non-invasive examination, it can be performed with OCT, and provide information field of view and OCT, which is conducive to early diagnosis of glaucoma, progress detection and risk assessment.

\subsection{Age-related Macular Degeneration}

Age-related macular degeneration (ARMD) is the main cause of central vision loss in the world. There are two main types: (1) atrophic (dry) age-related macular degeneration; (2) exudative (wet) age-related macular degeneration. Dry age-related macular degeneration is mainly caused by RPE degeneration, which is manifested by early macular depigmentation and proliferation, drusen, and late geographic atrophy [81]; exudative age-related macular degeneration is mainly the formation of CNV. The positional relationship with RPE can be divided into three types. Type I CNV originates from the choroid, mainly between Bruch's membrane and the retinal pigment epithelium; II CNV is located above the retinal pigment epithelium; and III CNV is a retinal inward, which is mixed by I and II $[82,83]$.

With the development of OCTA as a rapid and non-invasive fundus angiography technology, OCTA can provide deeper information on the structure and vascular of the retina and choroid [84], and improve our understanding of early retinal and choroidal diseases. First of all, SS-OCTA can be used in early ARMD patients to scan areas where there is no blood flow signal in the choroid capillary layer (flow gap) and areas where choroidal capillary perfusion is reduced, and this analysis method has high repeatability $[85,86]$. Mastropasqua et al. [87] found that the retinal vascular plexus density and choroidal thickness in patients with early ARMD were significantly lower than those in normal eyes. These findings provide the possibility for the application of OCTA in early ARMD screening [87]. Secondly, the visualization of choroidal vascular loss can be realized by SS-OCTA. OCTA can quantitatively analyze the blood flow and area of CNV in exudative AMD. Exudative ARMD is mainly manifested as choroidal neovascularization. OCTA can accurately identify the area of choroidal neovascularization, which is usually surrounded by the unperfused area of choroidal capillaries [ 43 , 88]. At the same time, it has the ability to quantify choroidal neovascularization, and the sensitivity ranges from $50 \%$ to $100 \%$. Furthermore, as a non-invasive fundus angiography technique, OCTA is beneficial to the observation of choroidal neovascularization before and after ARMD treatment [89].
Coscas et al. [90] reported the changes of CNV area before and after intravitreous injection of anti-vascular endothelial growth factor (VEGF). They found that the area of CNV decreased to some extent after anti-VEGF treatment, and the reduction of the area of II $\mathrm{CNV}$ is greater than that of I CNV. Perhaps it is because the anti-VEGF drugs are not easy to penetrate under the RPE layer, which leads to a better curative effect on II CNV. It can be seen that OCTA enhances our understanding of choroidal vessels and provides help for disease progression and treatment.

\subsection{High Myopia}

Myopia is the highest incidence of eye disease in the world, and the incidence has been increasing, which is a public health problem of global concern. High myopia (HM) is an important cause of vision loss, especially among young people. High myopia is defined as myopia with spherical equivalent $(\mathrm{SE}) \geq$ $-6.0 \mathrm{D}$ and (or) eye axis $\geq 26 \mathrm{~mm}$. It is an ophthalmic disease characterized by the gradual extension of the axial length of the eye and the gradual degeneration of the posterior pole [91, 92]. High myopia and its fundus complications seriously affect visual function.

With the advent of new techniques such as OCT and OCTA, our understanding of macular degeneration in myopia has been greatly improved. Studies have shown that the increase of diopter and axial length in myopia will lead to many degenerative changes in the retina, which may cause vision loss [93]. Therefore, in-depth research on retinal microangiopathy to understand the occurrence, development and prevention of high myopia has been the focus of scholars at home and abroad. Wang et al. [93] found that the blood flow density around the optic disc decreased in high myopia, which was negatively correlated with the axial length, and the retinal blood perfusion around the papilla was positively correlated with the thickness of the optic nerve fiber layer. These vascular characteristics may increase the probability of vascular related diseases in high myopia.

OCTA realizes the visualization of choroidal capillaries and choroidal vascular. Al-Sheikh et al. [94] used OCTA to study the retinal capillaries and choroidal capillaries in myopia. It was found that retinal capillary density decreased, choroidal capillary blood flow was deficiency and cavity area increased in high myopia. Wong et al. [95] also reached the same conclusion that with the aggravation of myopic maculopathy, choroidal capillary circulatory disorders also aggravated, which can turn from localized lesions to extensive. It is also found that within patients with high myopia and slight fundus changes (such as no obvious fundus atrophy of retinal pigment epithelium), the decrease of choroidal capillary vsacular density can also be observed.

For choroidal neovascular disease with high myopia, OCTA can detect abnormal structures of neovascularization at different levels and distinguish the state of CNV activity. For example, the active CNV is characterized by a typical lace wheel pattern, accompanied by small capillaries, mutually anastomosing grid-like structures, and a low-concentration 
halo around the lesion. Static CNV is characterized by long filamentous large mature blood vessels and dead tree-like morphology that coincide with each other. At the same time, OCTA is also highly sensitive to some neovascularization lesions. OCTA successfully detected the CNV detection rate of myopia as high as $94.1 \%$ [96]. Therefore, OCTA may help us to discover the potential pathophysiological characteristics of myopia and make it possible for early detection and prevention of myopic retinopathy.

\section{Conclusions}

OCTA has great potential in clinical applications. Because retinal microvessels have the same characteristics as cerebral blood vessels, researchers are studying the retina microvascular damage to further understand the microvascular damage in central nervous system degenerative diseases. OCTA has been applied to the detection of central nervous system diseases, for example, Alzheimer's disease, Parkinson's disease and multiple sclerosis. This is to evaluate whether OCTA can be used as an early diagnosis and monitoring of neurodegenerative diseases [97]. In some cases, it has even been proven that OCTA can detect pathological changes that are not seen on FFA. Due to the limitations of OCTA, such as small imaging range and motion artifacts, faster non-invasive scanning speed is very important to obtain a larger field of vision with higher resolution in the future. Higher and larger vision is essential. It is undeniable that because of the characteristics of non-invasive, rapid, good reproducibility and layered scanning of OCTA, clinical ophthalmologists have a deeper understanding of exploring the blood vessels of retinal diseases, which is helpful for us to understand the pathogenesis, diagnosis, treatment and follow-up of the disease. More research is needed in the future to determine the effectiveness of OCTA in the clinical environment.

\section{References}

[1] Schwartz D M, Fingler J, Kim D Y, et al. Phase-variance optical coherence tomography: a technique for noninvasive angiography [J]. Ophthalmology, 2014, 121 (1): 180-187.

[2] Kim D Y, Fingler J, Zawadzki R J, et al. Optical imaging of the chorioretinal vasculature in the living human eye $[\mathrm{J}]$. Proceedings of the National Academy of Sciences of the United States of America, 2013, 110 (35): 14354-14359.

[3] Choi W, Mohler K J, Potsaid B, et al. Choriocapillaris and choroidal microvasculature imaging with ultrahigh speed OCT angiography [J]. PloS one, 2013, 8 (12): e81499.

[4] Stanga P E, Tsamis E, Papayannis A, et al. Swept-Source Optical Coherence Tomography Angio ${ }^{\mathrm{TM}}$ (Topcon Corp, Japan): Technology Review [J]. Developments in ophthalmology, 2016, 56: 13-17.

[5] Gass J D M, Sever R J, Sparks D, et al. A Combined Technique of Fluorescein Funduscopy and Angiography of the Eye [J]. Archives of Ophthalmology, 1967, 78 (4): 455.
[6] Novotny H R, Alvis D L. A method of photographing fluorescence in circulating blood in the human retina $[\mathrm{J}]$. Circulation, 1961, 24: 82-86.

[7] Kogure K, Choromokos E. Infrared absorption angiography [J]. Journal of Applied Physiology, 1969, 26 (1): 154.

[8] Yannuzzi L A, Slakter J S, Sorenson J A, et al. Digital indocyanine green videoangiography and choroidal neovascularization [J]. Retina, 1992, 12 (3): 191-223.

[9] Staurenghi G, Bottoni F, Giani A. Clinical Applications of Diagnostic Indocyanine Green Angiography [J]. Retina, 2013, 1: $51-81$.

[10] Spaide R F, Klancnik J M, Cooney M J. Retinal Vascular Layers Imaged by Fluorescein Angiography and Optical Coherence Tomography Angiography [J]. Jama Ophthalmol, 2015, 133 (1): 45-50.

[11] Do D V. Detection of new-onset choroidal neovascularization [J]. Ophthalmology, 2013, 24 (3): 244-247.

[12] Do D V, Gower E W, Cassard S D, et al. Detection of New-Onset Choroidal Neovascularization Using Optical Coherence Tomography: the AMD DOC Study [J]. Ophthalmology, 2012, 119 (4): 771-778.

[13] Kotsolis A I, Killian F A, Ladas I D, et al. Fluorescein Angiography and Optical Coherence Tomography Concordance for Choroidal Neovascularization in Multifocal Choroiditis [J]. British Journal of Ophthalmology, 2010, 94 (11): 1506-1508.

[14] Johnson R N, Fu A D, Mcdonald H R, et al. Fluorescein Angiography: Basic Principles and Interpretation [J]. Retina, 2013, 1: 2-50.

[15] Bressler N M, Bressler S B. Chapter 66-Neovascular (Exudative or "Wet") Age-Related Macular Degeneration [J]. Retina: fifth Edition, 2013: 1183-1212.

[16] Kaiser P K. Chapter 53 - Branch Vein Occlusion [M]. Elsevier Inc., 2013.

[17] Lopez-Saez M P, Ordoqui E, Tornero $P$, et al. Fluorescein-Induced Allergic Reaction [J]. Ann Allergy Asthma Immunol, 1998, 81 (5): 428-430.

[18] Kwiterovich K A, Maguire M G, Murphy R P, et al. Frequency of adverse systemic reactions after fluorescein angiography. Results of a prospective study [J]. Ophthalmology, 1991, 98 (7): 1139-42.

[19] Carlo T E D, Romano A, Waheed N K, et al. A review of optical coherence tomography angiography (OCTA) [J]. International Journal of Retina Vitreous, 2015, 1: 5.

[20] Chua J, Chin C W L, Tan B, et al. Impact of systemic vascular risk factors on the choriocapillaris using optical coherence tomography angiography in patients with systemic hypertension [J]. Scientific Reports, 2019, 9 (1): 5819.

[21] Garrity S T, Iafe N A, Nopasak P, et al. Quantitative Analysis of Three Distinct Retinal Capillary Plexuses in Healthy Eyes Using Optical Coherence Tomography Angiography [J]. Invest Ophthalmol Vis Sci, 2017, 58 (12): 5548-5555.

[22] Guo Y, Camino A, Zhang M, et al. Automated segmentation of retinal layer boundaries and capillary plexuses in wide-field optical coherence tomographic angiography [J]. Biomedical Optics Express, 2018, 9 (9): 4429-4442. 
[23] Hwang T S, Zhang M, Bhavsar K, et al. Visualization of 3 Distinct Retinal Plexuses by Projection-Resolved Optical Coherence Tomography Angiography in Diabetic Retinopathy [J]. Jama Ophthalmology, 2016, 134 (12): 1411-1419.

[24] Matsunaga D, Yi J, Puliafito C A, et al. OCT Angiography in Healthy Human Subjects [J]. Ophthalmic Surg Lasers Imaging Retina, 2014, 45 (6): 510-515.

[25] Pichi F, Sarraf D, Morara M, et al. Pearls and pitfalls of optical coherence tomography angiography in the multimodal evaluation of uveitis $[\mathrm{J}]$. Journal of Ophthalmic Inflammation Infection, 2017, 7 (1): 20.

[26] Zhang Q, Lee C S, Chao J, et al. Wide-field optical coherence tomography based microangiography for retinal imaging $[\mathrm{J}]$. Scientific Reports, 2016, 6 (1): 22017.

[27] Nesper P L, Soetikno B T, Zhang H F, et al. OCT Angiography and Visible-Light OCT in Diabetic Retinopathy [J]. Vision Research, 2017, 139: 191-203.

[28] Sambhav K, Grover S, Chalam K V. The application of optical coherence tomography angiography in retinal diseases $[\mathrm{J}]$. Survey of Ophthalmology, 2017, 62 (6): 838-866.

[29] Lee R, Wong T Y, Sabanayagam C, et al. Epidemiology of diabetic retinopathy, diabetic macular edema and related vision loss [J]. Eye, 2015, 2 (1): 17.

[30] Saaddine J B, Honeycutt A A, Narayan K M, et al. Projection of diabetic retinopathy and other major eye diseases among people with diabetes mellitus: United States, 2005-2050 [J]. Arch Ophthalmol, 2008, 126 (12): 1740-1747.

[31] Klein K, Eden B. Overview of epidemiologic studies of diabetic retinopathy [J]. Ophthalmic Epidemiology, 2007, 14 (4): 179-183.

[32] Campochiaro, Peter A. Molecular Pathogenesis of Retinal and Choroidal Vascular Diseases [J]. Progress in Retinal Eye Research, 2015, 49: 67-81.

[33] Stitt AW, Curtis TM, Chen M, et al. The progress in understanding and treatment of diabetic retinopathy [J]. Progress in Retinal Eye Research, 2016, 51: 156-186.

[34] Ishibazawa A, Nagaoka T, Takahashi A, et al. Optical Coherence Tomography Angiography in Diabetic Retinopathy: A Prospective Pilot Study [J]. American Journal of Ophthalmology, 2015, 160 (1): 35-44.e1.

[35] Yu S, Lu J, Cao D, et al. The role of optical coherence tomography angiography in fundus vascular abnormalities $[\mathrm{J}]$. Bmc Ophthalmology, 2016, 16 (1): 1-7.

[36] Miwa Y, Murakami T, Suzuma K, et al. Relationship between Functional and Structural Changes in Diabetic Vessels in Optical Coherence Tomography Angiography [J]. Scientific Reports, 2016, 6: 29064.

[37] Peres M B, Kato R T, Kniggendorf V F, et al. Comparison of Optical Coherence Tomography Angiography and Fluorescein Angiography for the Identification of Retinal Vascular Changes in Eyes With Diabetic Macular Edema [J]. Ophthalmic Surgery Lasers Imaging Retina, 2016, 47 (11): 1013.

[38] Lee J, Moon B G, Cho A R, et al. Optical Coherence Tomography Angiography of DME and Its Association with Anti-VEGF Treatment Response [J]. Ophthalmology, 2016, 123 (11): 2368-2375.

[39] Jiang Y, Lim J, Degillio A. Profound Macular Ischemia on
Optical Coherence Tomography Angiography in Severe Diabetic Retinopathy [J]. Ophthalmology, 2017, 124 (6): 785 .

[40] Singh A, Agarwal A, Mahajan S, et al. Morphological differences between optic disc collaterals and neovascularization on optical coherence tomography angiography [J]. Graefes Archive for Clinical Experimental Ophthalmology, 2017, 255 (4): 753-759.

[41] Savastano M C, Federici M, Falsini B, et al. Detecting papillary neovascularization in proliferative diabetic retinopathy using optical coherence tomography angiography [J]. Acta ophthalmologica, 2018, 96 (3): 321-323.

[42] Falavarjani K G, Habibi A, Khorasani M A, et al. Time course of changes in optical disk neovascularization after a single intravitreal bevacizumab injection [J]. Retina, 2019, 39 (6): $1149-1153$

[43] Jia Y, Bailey S T, Hwang T S, et al. Quantitative optical coherence tomography angiography of vascular abnormalities in the living human eye [J]. Proc Natl Acad Sci USA, 2015, 112 (18): 2395-402.

[44] Freiberg F J, Pfau M, Wons J, et al. Optical coherence tomography angiography of the foveal avascular zone in diabetic retinopathy [J]. Graefes Archive for Clinical Experimental Ophthalmology, 2016, 254 (6): 1051-1058.

[45] Choi W J, Waheed N K, Moult E M, et al. Ultrahigh apeed swept source optical coherence tomography angiography of retinal and choriocapillaris alterations in diabetic patients with and without retinopathy [J]. Retina, 2017, 37 (1): 11-21.

[46] Carlo T E D, Filho M a B, Baumal C R, et al. Evaluation of Preretinal Neovascularization in Proliferative Diabetic Retinopathy Using Optical Coherence Tomography Angiography $[\mathrm{J}]$. Ophthalmic Surgery Lasers Imaging Retina, 2016, 47 (2): 115-119.

[47] Salz D A, De Carlo T E, Adhi M, et al. Select Features of Diabetic Retinopathy on Swept-Source Optical Coherence Tomographic Angiography Compared With Fluorescein Angiography and Normal Eyes [J]. Jama Ophthalmology, 2016, 134 (6): 644-650.

[48] Takase N, Nozaki M, Kato A, et al. Enlargement of foveal avascular zone in diabetic eyes fvaluated by en face optical coherence tomography angiography [J]. Retina, 2015, 35 (11): $2377-2383$

[49] Woo S C Y, Lip G Y H, Lip P L. Associations of retinal artery occlusion and retinal vein occlusion to mortality, stroke, and myocardial infarction: a systematic review [J]. Eye, 30 (8): $1031-1038$

[50] Hayreh S 2016S, Klugman M R, Beri M, et al. Differentiation of ischemic from non-ischemic central retinal vein occlusion during the early acute phase $[\mathrm{J}]$. Graefes Archive for Clinical Experimental Ophthalmology, 1990, 228 (3): 201-217.

[51] Group T C V O S. Baseline and early natural history report [J]. Arch Ophthalmol, 1993, 111 (8): 1087-95.

[52] Manuel C S, Anders K, Urban A, et al. Optical Coherence Tomography Angiography in Central Retinal Vein Occlusion: Correlation Between the Foveal Avascular Zone and Visual Acuity [J]. Invest Ophthalmol Vis Sci, 2016, 57 (9): OCT242. 
[53] Dan S, Fingler J, Werner J S, et al. Volumetric microvascular imaging of human retina using optical coherence tomography with a novel motion contrast technique [J]. Optics Express, 2009, 17 (24): 22190-200.

[54] Potsaid B, Huang D, Subhash H, et al. Split-spectrum amplitude-decorrelation angiography with optical coherence tomography [J]. Optics Express, 2012, 20 (4): 4710-4725.

[55] Kuehlewein L, An L, Durbin M K, et al. Imaging areas of retinal nonperfusion in ischemic branch retinal vein occlusion with swept-source OCT microangiography [J]. Ophthalmic Surgery Lasers Imaging Retina, 2015, 46 (2): 249.

[56] Suzuki N, Hirano Y, Yoshida M, et al. Microvascular Abnormalities on Optical Coherence Tomography Angiography in Macular Edema Associated With Branch Retinal Vein Occlusion [J]. American Journal of Ophthalmology, 2016, 161: 126-32.e1.

[57] Joo, Nobre, Cardoso, et al. Systematic Evaluation of Optical Coherence Tomography Angiography in Retinal Vein Occlusion [J]. American Journal of Ophthalmology, 2016, 163: 93-107.e6.

[58] Mehreen, Adhi, Marco, et al. Retinal Capillary Network and Foveal Avascular Zone in Eyes with Vein Occlusion and Fellow Eyes Analyzed With Optical Coherence Tomography Angiography [J]. Investigative Ophthalmology Visual Science, 2016, 57 (9): OCT486-94.

[59] Samara W A, Say E a T, Khoo C T L, et al. Correlation of foveal avascular zone size with foveal morphology in normal eyes using optical coherence tomography angiography [J]. Retina, 2015, 35 (11): 2188-2195.

[60] Mastropasqua R, Toto L, Antonio L D, et al. Correction: Corrigendum: Optical coherence tomography angiography microvascular findings in macular edema due to central and branch retinal vein occlusions [J]. Scientific Reports, 2017, 7: 40763.

[61] Koulisis N, Kim A Y, Chu Z, et al. Quantitative microvascular analysis of retinal venous occlusions by spectral domain optical coherence tomography angiography [J]. Plos One, 2017, 12 (4): e0176404.

[62] Laura, Kuehlewein, Tudor, et al. Noninvasive Visualization and Analysis of the Human Parafoveal Capillary Network Using Swept Source OCT Optical Microangiography [J]. Investigative Opthalmology Visual Science, 2015, 56 (6): 3984-3988.

[63] Carpineto P, Mastropasqua R, Marchini G, et al. Reproducibility and repeatability of foveal avascular zone measurements in healthy subjects by optical coherence tomography angiography [J]. Br J Ophthalmol, 2016, 100 (5): 671-676.

[64] Glacet-Bernard A, Sellam A, Coscas F, et al. Optical coherence tomography angiography in retinal vein occlusion treated with dexamethasone implant: a new test for follow-up evaluation [J]. European Journal of Ophthalmology, 2016, 26 (5): 460-468.

[65] Parodi M B, Visintin F, Rupe P D, et al. Foveal avascular zone in macular branch retinal vein occlusion [J]. International Ophthalmology, 1995, 19 (1): 25-28.

[66] Tham Y C, Li X, Wong T Y, et al. Global prevalence of glaucoma and projections of glaucoma burden through 2040: a systematic review and meta-analysis [J]. Ophthalmology, 2014 121 (11): 2081-2090.

[67] Nongpiur M E, Ku J Y, Aung T. Angle closure glaucoma: a mechanistic review $[\mathrm{J}]$. Current Opinion in Ophthalmology, 2011, 22 (2): 96-101.

[68] Zhu L, Zong Y, Yu J, et al. Reduced Retinal Vessel Density in Primary Angle Closure Glaucoma: A Quantitative Study Using Optical Coherence Tomography Angiography [J]. Journal of Glaucoma, 2018, 27 (4): 322-327.

[69] Yali, Jia, Eric, et al. Optical Coherence Tomography Angiography of Optic Disc Perfusion in Glaucoma [J]. Ophthalmology, 2014, 121 (7): 1322-1332.

[70] Liu L, Jia Y, Takusagawa H L, et al. Optical Coherence Tomography Angiography of the Peripapillary Retina in Glaucoma [J]. Jama Ophthalmol, 2015, 133 (9): 1045-52.

[71] Rao HL, Pradhan ZS, Weinreb R N, et al. Regional Comparisons of Optical Coherence Tomography Angiography Vessel Density in Primary Open-Angle Glaucoma [J]. American Journal of Ophthalmology, 2016, 171: 75-83.

[72] Rao H L, Pradhan Z S, Weinreb R N, et al. A comparison of the diagnostic ability of vessel density and structural measurements of optical coherence tomography in primary open angle glaucoma [J]. PloS one, 2017, 12 (3): e0173930.

[73] Takusagawa H L, Liu L, Ma K N, et al. Projection-Resolved Optical Coherence Tomography Angiography of Macular Retinal Circulation in Glaucoma [J]. Ophthalmology, 2017, 124 (11): 1589-1599.

[74] Suh MH, Zangwill LM, Manalastas PIC, et al. Deep Retinal Layer Microvasculature Dropout Detected by the Optical Coherence Tomography Angiography in Glaucoma [J]. Ophthalmology, 2016, 123 (12): 2509-2518.

[75] Ji LE, Tae-Woo K, Hyen L S, et al. Underlying Microstructure of Parapapillary Deep-Layer Capillary Dropout Identified by Optical Coherence Tomography Angiography [J]. Investigative Ophthalmology Visual Science, 2017, 58 (3): 1621.

[76] Shin JW, Kwon J, Lee J, et al. Choroidal Microvasculature Dropout is Not Associated With Myopia, But is Associated With Glaucoma [J]. Journal of Glaucoma, 2018, 27 (2): 189-196.

[77] Mansoori T, Sivaswamy J, Gamalapati J S, et al. Radial Peripapillary Capillary Density Measurement Using Optical Coherence Tomography Angiography in Early Glaucoma [J]. Journal of Glaucoma, 2017, 26 (5): 438-443.

[78] Scripsema N K, Garcia P M, Bavier R D, et al. Optical Coherence Tomography Angiography Analysis of Perfused Peripapillary Capillaries in Primary Open-Angle Glaucoma and Normal-Tension Glaucoma $[\mathrm{J}]$. Investigative Ophthalmology Visual Science, 2016, 57 (9): OCT611.

[79] Chieh-Li, Chen, Anqi, et al. Peripapillary Retinal Nerve Fiber Layer Vascular Microcirculation in Glaucoma Using Optical Coherence Tomography-Based Microangiography [J]. Investigative Ophthalmology Visual Science, 2016, 57 (9): OCT475-85.

[80] Hou H, Moghimi S, Zangwill L M, et al. Macula Vessel Density and Thickness in Early Primary Open-Angle Glaucoma [J]. American Journal of Ophthalmology, 2019, 199: 120-132. 
[81] Jeffrey, Ma, Ria, et al. Optical Coherence Tomographic Angiography Imaging in Age-Related Macular Degeneration [J]. Ophthalmology Eye Diseases, 2017, 9: 1179.

[82] Spaide R F, Fujimoto J G, Waheed N K, et al. Optical coherence tomography angiography [J]. Progress in Retinal Eye Research, 2018, 64: 1-55.

[83] Arya M, Sabrosa A S, Duker J S, et al. Choriocapillaris changes in dry age-related macular degeneration and geographic atrophy: a review [J]. Eye Vision, 2018, 5 (1): 22.

[84] Zheng F, Zhang Q, Shi Y, et al. Age-Dependent Changes in the Macular Choriocapillaris of Normal Eyes Imaged with Swept-Source OCT Angiography [J]. American Journal of Ophthalmology, 2019, 200: 110-122.

[85] Zhang Q, Fang Z, Motulsky E H, et al. A Novel Strategy for Quantifying Choriocapillaris Flow Voids Using Swept-Source OCT Angiography [J]. Investigative Ophthalmology Visual Science, 2018, 59 (1): 203-211.

[86] Waheed N K, Moult E M, Fujimoto J G, et al. Optical Coherence Tomography Angiography of Dry Age-Related Macular Degeneration [J]. Developments in ophthalmology, 2016, 56: 91-100.

[87] Toto L, Borrelli E, Antonio L D, et al. Retinal vascular plexuses? changes in dry age-related macular degeneration, evaluated by means of optical coherence tomography angiography [J]. Retina, 2016, 36 (8): 1566-1572.

[88] Talisa, E., De, et al. Spectral-Domain Optical Coherence Tomography Angiography of Choroidal Neovascularization [J]. Ophthalmology, 2015, 122 (6): 1228-38.

[89] Miere A, Querques G, Semoun O, et al. Optical coherence tomography angiography in early type 3 neovascularization $[\mathrm{J}]$. Retina, 2015, 35 (11): 2236-2241.
[90] Coscas G, Lupidi M, Coscas F, et al. Optical Coherence Tomography Angiography during Follow-Up: Qualitative and Quantitative Analysis of Mixed Type I and II Choroidal Neovascularization after Vascular Endothelial Growth Factor Trap Therapy [J]. Ophthalmic Research, 2015, 54 (2): 57-63.

[91] Morgan I G, Ohno-Matsui K, Saw S-M. Myopia [J]. Lancet, 2012, 379 (9827): 1739-1748.

[92] Miller D G, Singerman L J. Natural history of choroidal neovascularization in high myopia [J]. Current opinion in ophthalmology, 2001, 12 (3): 222-224.

[93] Wang X, Kong X, Jiang C, et al. Is the peripapillary retinal perfusion related to myopia in healthy eyes? A prospective comparative study [J]. Bmj Open, 2016, 6 (3): e010791.

[94] Mayss A S, Nopasak P, Rosa D M, et al. Quantitative OCT Angiography of the Retinal Microvasculature and the Choriocapillaris in Myopic Eyes [J]. Invest Ophthalmol Vis Sci, 2017, 58 (4): 2063.

[95] Wong CW, Teo YCK, Tsai STA, et al. Characterization of the choroidal vasculature in myopic maculopathy with optical coherence tomographic angiography [J]. Retina, 2019, 39 (9): 1742-1750.

[96] Miyata M, Ooto S, Hata M, et al. Detection of Myopic Choroidal Neovascularization Using Optical Coherence Tomography Angiography [J]. American Journal of Ophthalmology, 2016, 165: 108-114.

[97] Pellegrini M, Vagge A, Ferro Desideri L F, et al. Optical Coherence Tomography Angiography in Neurodegenerative Disorders [J]. Journal of clinical medicine, 2020, 9 (6): 1706. 